\title{
XXIII. Ausgleichungsmethode der geometrischen Krystallographie.
}

Von

Carl Viola in Rom.

(Mit 5 Textfiguren.)

Wăhrend einerseits die Beobachtungsmittel immer grössere Genauigkeit erlangen, fasst anderseits die Theorie der kleinsten Quadrate Boden in der Krystallographie, und diese letztere wird zu einer Präcisionswissenschaft erhoben. Bei uns zwar bedienen sich die Krystallographen noch der alten Methoden zur Berechnung der Axenelemente eines Krystalles, d. h. derjenigen Methoden, welche, ohne die Beobachtungsfehler in Betracht zu ziehen, rasch zu Ergebnissen führen, die fruher allenfalls annehmbar waren. Aber heutzutage, wo die physikalischen Experimente so grosse Fortschritte gemacht haben, sei es was die Wärmeausdehnung anbelangt, sei es was die Elasticităt oder andere Präcisionsuntersuchungen der Krystalle anbetrifft, macht sich die Nothwendigkeit fublbarer, die wahrscheinlichsten idealen Grundelemente der Krystalle zu bestimmen. Zu diesem Zwecke zieht man sich die Winkel zu Nutzen, welche zur Verfügung stehen oder zur Verfugung gestellt werden können.

Schon J. Beckenkamp*) berechnete im Jahre 1881 in seiner Inauguraldissertation die Axenelemente nach der Methode der Ausgleichungsrechnung; nachber**) stellte er die auf die Theorie der kleinsten Quadrate sich stützende Ausgleichungsrechnung in sehr einfacher und klarer Weise dar, so dass die in der Algebra weniger geibbten Krystallographen nur den von ihm vorgeschlagenen Rechnungsgang zu verfolgen brauchen.

Die Ausgleichungsrechnung kann auch eine von der gewöhnlich angewandten verschiedene Form annehmen, ich möchte sagen eine elegantere, theilweise logarithmische und deshalb auch einfachere Form; aber um

*) Diese Zeilschr. 5, 436.

**) Diese Zeitschr. 22, 376. 
dahin zu gelangen, muss man von Betrachtungen ausgehen, welche nicht zu der Theorie der kleinsten Quadrate gebören, wohl aber zu demselben Ziele führen wie diese.

Dieser Aufsatz hat den Zweck; dem Leser diese Methode mitzutheilen.

Vergegenwärtigen wir uns zuvörderst kurz die Aufgabe, um die es sich handell. Man hat alle sechs Winkel von vier Grundflächen eines Krystalles gemessen, und man will in der wahrscheinlichsten Weise diese sechs Winkel corrigiren, jedoch so, dass der von den vier Flächen zusammengesetzte Körper im Raume möglich sei. Die gemessenen Winkel zwischen den Polaren der vier Flächen sind $a_{1}, a_{2}, a_{3}, a_{4}, a_{5}, a_{6}$, ihre Gewichte oder

Fig 1.

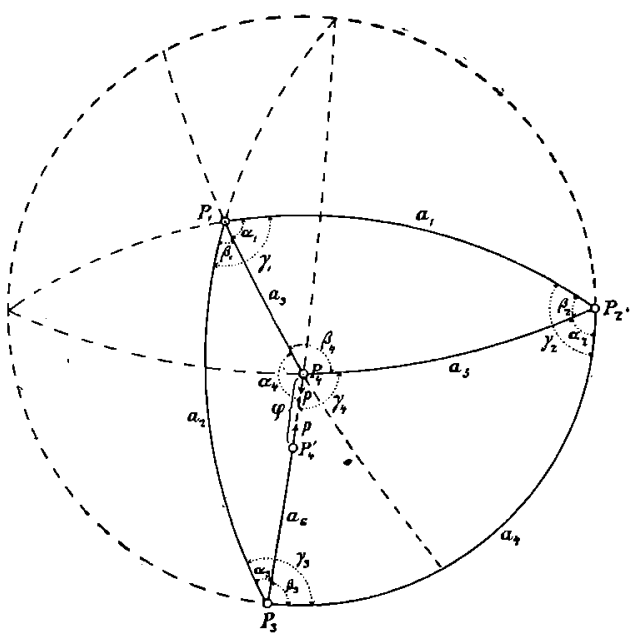
Genauigkeilmaasse sind $g_{1}, g_{2}$, $g_{3}, g_{4}, g_{5}, g_{6}$. Um diese Aufgabe klarer darzustellen, kann man sie in eine grapbische For'm bringen. $\mathrm{Zu}$ diesem Zwecke construiren wir, Fig. 1 , auf der spbärischen Oberfläche, deren Radius 1 ist, die zu den vier Flächen gehörenden Pole $P_{1}, P_{2}, P_{3}, P_{4}$ vermiltelst der ersten fünf Winkel $a_{1}, a_{2}, a_{3}$, $a_{4}, a_{5}$, so wie sie durch Messung mit dem Goniometer erhalten werden. Wenn man nun die Zone $P_{3} P_{4}$ betrachtet, so wird der so erhaltene Winkel $\widetilde{P_{3} P_{4}}$, den wir $a_{6}{ }^{\prime}$ nennen wollen, nicht gleich dem gemessenen Winkel $a_{6}$ sein, ausser in zufäligen und somit unwahrscheinlichsten Fällen. Wir nehmen nalurlich an, dass sie nicht gleich seien, und bezeichnen die Differenz beider als den Widerspruch des Systems:

$$
\varphi=a_{6}{ }^{\prime}-a_{6} .
$$

Wenn wir also den Winkel $a_{6}$ von dem Pole $P_{3}$ aus auftragen, so werden wir einen fünften Pol $P_{4}{ }^{\prime}$ erhalten. Die Aufgabe ist somit folgende : Den Winkeln $a_{1}, a_{2}, a_{3}, a_{4}, a_{5}, a_{6}$ gebe man solche Verbesserungen $c_{1}, c_{2}$, $c_{3}, c_{4}, c_{5}, c_{6}$, dass die zwei Pole $\boldsymbol{P}_{4}$ und $\boldsymbol{P}_{4}^{\prime}$ sich decken. Die Lösung dieser Aufgabe ist naturlich auf viele Weisen möglich, weil unendlich viele Systeme $c_{1}, c_{2}, c_{3}, c_{4}, c_{5}, c_{6}$ ihr entsprechen können. Wenn man aber das System von $c_{1} c_{2} \ldots c_{6}$, welches der Theorie der kleinsten Quadrate entspricht, nämlich :

1) $g_{1} c_{1}{ }^{2}+g_{2} c_{2}{ }^{2}+g_{3} c_{3}{ }^{2}+g_{4} c_{4}{ }^{2}+g_{5} c_{5}{ }^{2}+g_{6} c_{6}{ }^{2}=$ Min., wählen will, so wissen wir, dass die Aufgahe eindeutig bestimmt ist. 
Um zu einer einfachen Lösung zu gelangen, wollen wir sehen, ob der Aufgabe eine mechanische Bedeutung zu Grunde liegt.

Wenn die Winkel $a_{1}, a_{2} \ldots \ldots a_{6}$ dureh innere, in den respectiven Zonen sich zu denkende Kräfte veränderlich wären, und man durch zwei an die zwei Polaren $P_{4}$ und $P_{4}{ }^{\prime}$ angebrachte Kräftepaare versuchte, die eben erwähnten Polaren zu nähern, bis sie sich decken, so wüde der Zweck vollkommen erreicht sein, da jeder Winkel einen Zuwachs (Abnahme = negativer Zuwachs) erhalten wurde, und zwar in dem Maasse, als die inneren sich entwickelnden Kräftepaare den äusseren das Gleichgewicht hielten. Es handelt sich nun darum zu sehen, wie die inneren Kräfte beschaffen sein mussen, damit die Veränderungen, welche die Winkel erlitten haben, als Correctionen angesehen, dem Principe der kleinsten Quadrate Genuge leisten. Es ist sofort klar, dass, wenn die inneren Kräftepaare den Werthen

$$
g_{1} c_{1}, \quad g_{2} c_{2}, \quad g_{3} c_{3}, \quad g_{4} c_{4}, \quad g_{5} c_{5}, \quad g_{6} c_{6}
$$

gleich gesetzt werden, die Forderungen erfullt sind. In der That, wenn wir mit $p$ eines der äusseren angewandten Kräftepaare und mit $\delta \varphi$ die sehr kleine Winkeldrehung bezeichnen, welche die Polare $P_{4}{ }^{\prime}$ gegen die Polare $P_{4}$ und in Bezug auf dieselbe ausfuhrt, so werden wir folgende Gleichung erhalten:

2) $p \delta \varphi=g_{1} c_{1} \delta c_{1}+g_{2} c_{2} \delta c_{2}+g_{3} c_{3} \delta c_{3}+g_{4} c_{4} \delta c_{4}+g_{5} c_{5} \delta c_{5}+g_{6} c_{6} \delta c_{6}$, wobei $\delta c_{1}, \delta c_{2} \ldots \ldots \delta c_{6}$ die unendlich kleinen Winkelveränderungen bedeuten, da die äussere Arbeit derjenigen der inneren Kräfte jeden Augenblick gleichkommt.

Und nun, wenn mit Hulfe des Kräftepaares $p$ die Winkelveränderungen so weit vorgeschritten sind, dass das Gleichgewicht eintritt, so besteht $p \delta \varphi=0$, und somit werden wir erhalten :

$$
g_{1} c_{1} \delta c_{1}+g_{2} c_{2} \delta c_{2}+g_{3} c_{3} \delta c_{3}+g_{4} c_{4} \delta c_{4}+g_{5} c_{5} \delta c_{5}+g_{6} c_{6} \delta c_{6}=0,
$$

was in der That mit Gleichung 1) ubereinstimmt.

Daraus folgt: Das einer Bedingungsgleichung unterworfene Problem der Ausgleichung der Beobachtungsfehler kann dadurch gelöst werden, dass man das Gleichgewicht eines äusseren Kräften oder Kräfteparenausgesetzten elastischen Systems löst.

Indem wir die Gleichung 2) in Bezug auf $\varphi$ von 0 bis $\varphi$ integriren, wo $\varphi$ den Widerspruch $\widetilde{P_{4} P_{4}^{\prime}}$ bedeutet, erhalten wir:

3) $\quad 2 p \varphi=g_{1} c_{1}{ }^{2}+g_{2} c_{2}{ }^{2}+g_{3} c_{3}{ }^{2}+g_{4} c_{4}{ }^{2}+g_{5} c_{5}{ }^{2}+g_{6} c_{6}{ }^{2}$.

Hier haben wir eine kurze Bemerkung zu machen. Wenn man, um die Deformation des Systems zu erhalten, zwei Krältepaare von endlicher Grösse anwenden wurde, so wäre es unmöglich, das Gleichgewicht zu 
erhalten, da das System in Schwingungen geriethe. Wir müssen daher das als elastisch gedachte System so verändern, dass wir die Kraft $p$ nach und nach vergrössern, und zwar im Verhältniss zu der Abnahme des Widerspruches $\varphi$. Auf diese Weise wäre die äussere Arbeit nicht $p \varphi$, sondern $\frac{p \varphi}{2}$. Folglich müssen wir anstatt der Gleichung 3) folgende benutzen:

4) $\quad p \varphi=g_{1} c_{1}{ }^{2}+g_{2} c_{2}{ }^{2}+g_{3} c_{3}{ }^{2}+g_{4} c_{4}{ }^{2}+g_{5} c_{5}{ }^{2}+g_{6} c_{6}{ }^{2}$.

Verweilen wir dabei einen Augenblick, um zu sehen, wie sich die äusseren und die inneren Kräftepaare graphisch darstellen lassen. In jeder der secbs Zonen stellen wir uns zwei innere Kräftepaare vor, welche sich gegenseitig das Gleichgewicht halten. Da jedes Kräftepaar durch zwei gleiche, parallele und entgegengeselzte Kräfte ersetzt werden kann, so denken wir uns, dass jedes derselben in zwei gleiche, parallele und entgegengesetzte Kräfte zerlegt sei, und zwar so, dass die Kräfte in der Entfernung 1 , und in der betreffenden Zone liegen, und sie deshalb die bezưglichen Werthe

$$
g_{1} c_{1}, \quad g_{2} c_{2}, \quad g_{3} c_{3}, \quad g_{4} c_{4}, \quad g_{5} c_{5}, \quad g_{6} c_{6}
$$

haben. Eine der Kräfte jedes Paares geht durch den Kugelmittelpunkt und die andere ist tangential. So z. B. wirken in der Zone $\boldsymbol{P}_{1} \boldsymbol{P}_{2}$ zwei Kräftepaare, welche den Winkel $a_{1}$ zu vergrössern (verkleinern) streben. Diese beiden Kräftepaare sind gleich, und der Werth ihres Momentes ist $g_{1} c_{1} \times 1$. Man kann sich vorstellen, dass die eine der Kräfte, deren Werth $g_{1} c_{1}$ ist, in $P_{1}$ und die andere in $P_{2}$ angebracht sei.

Fig. 2.

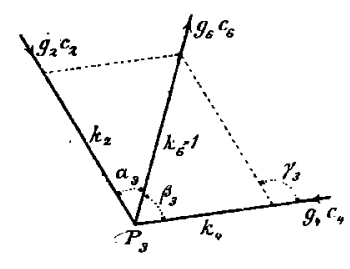

Da sich dieses System von Kräftepaaren das Gleichgewicht hält, gehen wir dazu über, letzteres in Bezug auf jeden Pol zu bestimmen. Im Pole $\boldsymbol{P}_{3}$, Fig. 2, wirken drei Kräfte, nämlich $g_{2} c_{2}$, $g_{4} c_{4}, g_{6} c_{6}$. Das Gleichgewicht dieser Kräfte verlangt:

$$
\begin{aligned}
& g_{2} c_{2}=g_{6} c_{6} \frac{\sin \beta_{3}}{\sin \gamma_{3}}=k_{2} \cdot g_{6} c_{6} \\
& g_{4} c_{4}=g_{6} c_{6} \frac{\sin \alpha_{3}}{\sin \gamma_{3}}=k_{4} \cdot g_{6} c_{6},
\end{aligned}
$$

was wir auch so schreiben können :

$$
\begin{aligned}
& g_{2} c_{2}{ }^{2}=\frac{k_{2}{ }^{2}}{g_{2}} \cdot g_{6}{ }^{2} c_{6}{ }^{2} \\
& g_{4} c_{4}{ }^{2}=\frac{k_{4}{ }^{2}}{g_{4}} \cdot g_{6}{ }^{2} c_{6}{ }^{2} .
\end{aligned}
$$

Weil auch der Pol $\boldsymbol{P}_{4}{ }^{\prime}$ im Gleichgewicht bleibt unter der Einwirkung der Kraft $g_{6} c_{6}$ und der ausseren Kraft $p$, ergiebt sich

$$
p=g_{6} c_{6} \text {. }
$$


Das Gleichgewicht des Poles $P_{\mathbf{f}}$ wird erhalten mit Hülfe der drei Kräfte

$$
p=g_{0} c_{6}, \quad g_{5} c_{5}, \quad g_{3} c_{3}
$$

und folglich muss, Fig. 3 :

$$
\begin{array}{lll}
g_{3} c_{3}=\frac{\sin \gamma_{4}}{\sin \beta_{4}} \cdot g_{6} c_{6}=k_{3} \cdot g_{6} c_{6} \quad \text { oder } & g_{3} c_{3}{ }^{2}=\frac{k_{3}{ }^{2}}{g_{3}} \cdot g_{6}{ }^{2} c_{6}{ }^{2} \\
g_{5} c_{5}=\frac{\sin \alpha_{4}}{\sin \beta_{4}} \cdot g_{6} c_{6}=k_{5} \cdot g_{6} c_{6} \quad-\quad g_{5} c_{5}{ }^{2}=\frac{k_{5}{ }^{2}}{g_{5}} \cdot g_{6}{ }^{2} c_{6}{ }^{2}
\end{array}
$$
sein.

Fig. 3.

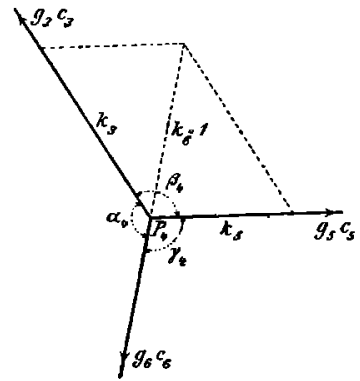

Fig. 4 .

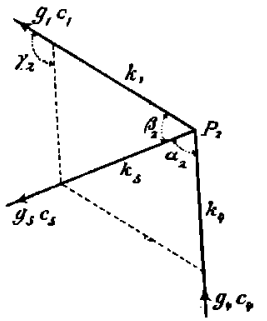

Schliesslich wirken im Pole $P_{2}$ die das Gleichgewicht haltenden Kräfte $g_{1} c_{1}, g_{4} c_{4}, g_{5} c_{5}$, und somit, Fig. 4,

$$
g_{1} c_{1}=\frac{\sin \alpha_{2}}{\sin \gamma_{2}} \cdot g_{5} c_{5}=\frac{\sin \alpha_{2}}{\sin \gamma_{2}} \cdot k_{5} g_{6} c_{6}=k_{1} g_{6} c_{6}
$$

oder

$$
g_{1} c_{1}^{2}=\frac{k_{1}^{2}}{g_{1}} \cdot g_{6}{ }^{2} c_{6}^{2} .
$$

Wenn wir die so erhallenen Werthe in die Gleichung 4) einsetzen, erbält dieselbe die Form :

$$
\varphi=\left[\frac{k_{1}^{2}}{g_{1}}+\frac{k_{2}^{2}}{g_{2}}+\frac{k_{3}^{2}}{g_{3}}+\frac{k_{4}^{2}}{g_{4}}+\frac{k_{5}^{2}}{g_{5}}+\frac{1}{g_{6}}\right] g_{6}{ }^{2} c_{6}{ }^{2},
$$

oder durch Gleichsetzung

$$
A=\frac{k_{1}^{2}}{g_{1}}+\frac{k_{2}^{2}}{g_{2}}+\frac{k_{3}^{2}}{g_{3}}+\frac{k_{4}^{2}}{g_{4}}+\frac{k_{5}^{2}}{g_{5}}+\frac{1}{g_{6}},
$$

7) die Form:

$$
g_{6} c_{6}=\frac{\varphi}{A} \text {. }
$$

\section{Zusammonfassung.}

Der Gang der Ausgleichung der beobachteten sechs Winkel zwischen vier Flächen eines beliebigen Krystalles, von welchem nicht drei Flachen derselben Zone angehören, ist demnach folgender: 
1) Man setze :

$$
\begin{array}{ll}
s_{1}=\frac{a_{1}+a_{3}+a_{5}}{2}, & s_{1}{ }^{\prime}=\frac{a_{1}+a_{3}-a_{5}}{2}, \\
s_{3}=\frac{a_{1}+a_{2}+a_{4}}{2}, & s_{3}{ }^{\prime}=\frac{a_{1}+a_{2}-a_{4}}{2}, \\
p_{1}=\sin s_{1} \cdot \sin s_{1}{ }^{\prime}, & \pi_{1}=\sin a_{1} \cdot \sin a_{3}, \\
p_{3}=\sin s_{3} \cdot \sin s_{3}{ }^{\prime}, & \pi_{3}=\sin a_{1} \cdot \sin a_{3} .
\end{array}
$$

2) $\quad \cos \frac{\alpha_{1}}{2}=\sqrt{\frac{p_{1}}{\pi_{1}}} \quad \cos \frac{\gamma_{1}}{2}=\sqrt{\frac{p_{3}}{\pi_{3}}}, \quad \beta_{1}=\gamma_{1}-\alpha_{1}$.

3) $\quad \cos a_{6}{ }^{\prime}=\cos a_{2} \cos a_{3}+\sin a_{2} \sin a_{3} \cos \beta_{1}$.

4) $\quad \sin \alpha_{3}=\sin \beta_{1} \cdot \frac{\sin a_{3}}{\sin a_{6}{ }^{\prime}}$.

5) $\quad \sin \alpha_{4}=\sin \beta_{1} \cdot \frac{\sin a_{2}}{\sin {a_{6}}^{\prime}}, \sin \beta_{4}=\sin \alpha_{1} \cdot \frac{\sin a_{1}}{\sin a_{5}}$.

6) $\quad \gamma_{4}=360^{0}-\left(\alpha_{4}+\beta_{4}\right)$.

7)

8) $\quad k_{1}=\frac{\sin \alpha_{2}}{\sin \gamma_{2}} \cdot \frac{\sin \alpha_{4}}{\sin \beta_{4}}$

$$
\begin{aligned}
& k_{2}=\frac{\sin \beta_{3}}{\sin \gamma_{3}} \\
& k_{3}=\frac{\sin \gamma_{4}}{\sin \beta_{4}} \\
& k_{4}=\frac{\sin \alpha_{3}}{\sin \gamma_{3}} \\
& k_{5}=\frac{\sin \alpha_{4}}{\sin \beta_{4}} \\
& k_{0}=1 .
\end{aligned}
$$

$$
A=\frac{k_{1}^{2}}{g_{1}}+\frac{k_{2}^{2}}{g_{2}}+\frac{k_{3}^{2}}{g_{3}}+\frac{k_{4}^{2}}{g_{4}}+\frac{k_{5}^{2}}{g_{5}}+\frac{k_{6}^{2}}{g_{6}} .
$$

10) $\quad g_{6} c_{6}=\frac{\varphi}{A}$.

11) $\quad g_{r} c_{r}=k_{r} \cdot g_{6} c_{6}$.

Das soeben berechnete Problem ist allgemein und bezieht sich naturlich auf das trikline System; es ist aber leicht auf specielle Fälle zu ubertragen. In dem monoklinen Systeme wird man z. B. zu setzen haben: $a_{2}=$ $a_{4}=90^{\circ}$ und somit $\gamma_{1}=\gamma_{2}=90^{\circ}$. Im rhombischen wird $a_{1}=a_{2}=$ $a_{4}=90^{\circ}$ sein und folglich $\gamma_{1}=\gamma_{2}=\gamma_{3}=90^{\circ} ; \mathrm{im}$ tetragonalen Systeme wird ausserdem $\alpha_{1}=\beta_{1}=45^{0}$ sein u. s. w. 
B e is piel.

Als Beispiel wähle ich das schon von J. Beckenkamp*) für das trikline System berechnele. Für Anorthit vom Vesuv hatte er folgende Winkel gefunden :

$$
\begin{array}{lll}
P_{1}: P_{2}=(11 T):(010)=a_{1}=115012^{\prime} 0,4 ; & g_{1}=1 \\
P_{1}: P_{3}=(11 T):(1 T 0)=a_{2}=98332,7 ; & g_{2}=1 \\
P_{1}: P_{4}=(11 T):(001)=a_{3}=574957,6 ; & g_{3}=1 \\
P_{2}: P_{3}=(010):(1 T 0)=a_{4}=1173416,4 ; & g_{4}=1 \\
P_{2}: P_{4}=(010):(001)=a_{5}=855417,4 ; & g_{5}=1 \\
P_{3}: P_{4}=(1 T 0):(001)=a_{6}=69200,8 ; & g_{6}=1
\end{array}
$$

Daraus berechnet sich :

$$
\begin{array}{ll}
s_{1}=129^{0} 28^{\prime} 77^{\prime \prime 7}, & s_{1}{ }^{\prime}=43^{0} 33^{\prime} 53^{\prime \prime 3}, 3 \\
s_{3}=165.3939,7, & s_{3}^{\prime}=48 \quad 523,3 .
\end{array}
$$

$\log p_{1}=9,725923, \quad \log p_{3}=9,265538$,

$\log \pi_{1}=9,884191, \quad \log \pi_{3}=9,951711$,

$\log \cos \frac{\alpha_{1}}{2}=9,920866, \quad \log \cos \frac{\gamma_{1}}{2}=9,656914$,

$$
\alpha_{1}=67^{\circ} 5^{\prime} 44^{\prime \prime}, 4, \quad \gamma_{1}=126^{0} 1^{\prime} 4^{\prime \prime}, 3
$$

$$
\begin{aligned}
\beta_{1} & =\gamma_{1}-\alpha_{1}=58^{0} 55^{\prime} 19^{\prime \prime}, 9, \\
\log \sin \beta_{4} & =9,929009, \quad \beta_{4}=123^{0} 19^{\prime} 10^{\prime \prime} 0, \\
\log \sin \alpha_{4} & =9,956746, \quad \alpha_{4}=115 \quad 858,0, \\
\gamma_{4} & =360^{0}-\left(\beta_{4}+\alpha_{4}\right)=121^{0} 31^{\prime} 52^{\prime \prime} 0 . \\
a_{6}{ }^{\prime} & =69^{0} 19^{\prime} 56^{\prime \prime}, 4, \\
\varphi & =a_{6}{ }^{\prime}-a_{6}=-4^{\prime \prime}, 4 .
\end{aligned}
$$

$\log \sin \alpha_{3}=9,889227, \quad \alpha_{3}=50047^{\prime} 34^{\prime \prime \prime}, 7$, $\log \sin \beta_{4}=9,981864, \quad \beta_{3}=733324,3$.

$$
\gamma_{4}=\beta_{3}+\alpha_{3}=124020^{\prime} 59^{\prime \prime}, 0 \text {. }
$$

$$
\begin{array}{ll}
\log k_{1}=0,033464_{n}, & k_{1}^{2}=1,16662 \\
\log k_{2}=0,065099_{n}, & k_{2}^{2}=1,34952 \\
\log k_{3}=0,008613, & k_{3}^{2}=1,04046 \\
\log k_{4}=9,972453_{n}, & k_{4}^{2}=0,88085 \\
\log k_{5}=0,034737, & k_{5}^{2}=1,17348 \\
& k_{6}^{2}=1,00000 \\
\hline A=6,61093
\end{array}
$$

$$
\begin{aligned}
& c_{6}=\frac{\varphi}{A}=-0,65 \\
& c_{1}=k_{1} c_{6}=+0,70 \\
& c_{2}=k_{2} c_{6}=+0,77
\end{aligned}
$$

*) Diese Zeitschr. 5, 442 und 22, 384 . 


$$
\begin{aligned}
& c_{3}=k_{3} c_{6}=-0,68 \\
& c_{4}=k_{4} c_{6}=+0,62 \\
& c_{5}=k_{5} c_{6}=-0,72 .
\end{aligned}
$$

Die Einfachheit dieser von mir vorgeschlagenen und zusammengeselzten Methode beruht in erster Linie darauf, dass die Berechnung der Coefficienten $k_{1} k_{2} k_{3} k_{4} k_{5}$ logarithmisch ist und auch graphisch sein kann; in zweiter Linie aber auch auf der Berechnung der Verbesserungen $c_{1} c_{2} c_{3} c_{4} c_{5} c_{6}$, indem man direct die Werthe in Bruchtheilen der Grade und nicht in solchen des Radius erhält, was eińen grossen Vortheil bietet.

Der Leser wird bemerkt haben, dass die Berechnung mil einer kleinen Ungenauigkeit behaftel ist, die jch absichtlich und stillschweigend ubergangen habe. Es wurden nämlich in der Berechnung von $k_{1} k_{2} k_{3} k_{4} k_{5}$ die Veränderungen nicht beachtet, welche die Winkel $\alpha_{1} \beta_{1} \gamma_{1}, \alpha_{3} \beta_{3} \gamma_{3}$ und $\alpha_{4} \beta_{4} \gamma_{4}$ erfahren in Folge der Zunahme $c_{1} c_{2} \ldots c_{5}$, welche die Winkel $a_{1} a_{2} \ldots a_{5}$ erhalten. Jedoch wird sich der Leser auch uberzeugen können, dass diese Veränderungen höchstens Fehler des zweiten Grades erzeugen, wenn wir annehmen, dass die Verbesserungen $c_{1} c_{2} \ldots c_{6}$ kleine Grössen des ersten Grades sind. So muss man eigentlich immer verfahren, wenn es sich um die Ausgleichungsrechnung nach der Methode der kleinsten Quadrate handelt, und die zu berechnenden Unbekannten in transcendentaler Form vorkommen. Für unseren $Z$ weck brauchen wir eine grössere Genauigkeit nicht zu verlangen.

Fig. 5.

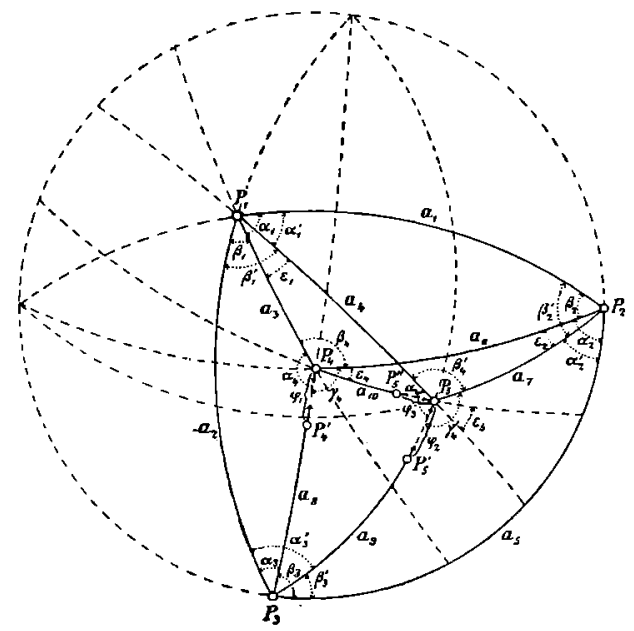

Dieser Vorgang ist auch auf einen allgemeinen Fall übertragbar, wenn die Beobachtungsfehler nämlich ,zwischen mehr als vier Flächen ausgeglichen werden sollen. Um dies klar darzulegen genugt es, dass wir funf solche in Betracht ziehen, zwischen denen man die Winkel $a_{1} a_{2} \ldots a_{10}$ gemessen hat; die Gewichte dieser Ergebnisse sind $g_{1} g_{2} \ldots$ $g_{10}$, und die Verbesserungen, die man an den Winkeln anzubringen hat, seien $c_{1} c_{2} \ldots c_{10}$. Construiren wir, Fig. 5, wie wir vorher gethan haben, die

Pole $P_{1} P_{2} P_{3} P_{4} P_{5}$ mit den irrthümlichen Winkeln $a_{1} a_{2} a_{3} a_{4} a_{5} a_{6} a_{7}$, so wird man die Winkel

$$
\widetilde{P_{3} P_{4}}=a_{8}^{\prime}, \quad \widetilde{P_{3} P_{5}}=a_{9}^{\prime} \quad \text { und } \widetilde{P_{4} P_{5}}=a_{10}^{\prime}
$$


erhallen, die verschieden sind von den gemessenen Winkeln $a_{8}, a_{9}, a_{10}$, sodass, wenn wir von $P_{3}$ aus gegen $P_{4}$ den Winkel $a_{8}$ auftragen, wir einen Pol $\boldsymbol{P}_{4}{ }^{\prime}$ erhalten, von $\boldsymbol{P}_{3}$ aus gegen $\boldsymbol{P}_{5}$ den Winkel $a_{9}$, einen Pol $\boldsymbol{P}_{5}{ }^{\prime}$, ferner von $P_{4}$ aus gegen $P_{5}$ denjenigen $a_{10}$, einen Pol $P_{5}^{\prime \prime}$. Die von einander unabhängigen Widersprüche des Systems werden sein :

$$
\begin{aligned}
& a_{8}{ }^{\prime}-a_{8}=\varphi_{1} \\
& a_{9}^{\prime}-a_{9}=\varphi_{2} \\
& a_{10}{ }^{\prime}-a_{10}=\varphi_{3} .
\end{aligned}
$$

Es handelt sich nun darum, ein solches System von Verbesserungen zu wählen, dass die drei oben erwähnten Widersprüche Null werden, und dass dem Princip der kleinsten Quadrate Genüge geleistet sei. Kommen wir auf die schon benutzte mechanische Bedeutung des Problems zurúck, so haben wir in den Zonen $P_{4} P_{4}{ }^{\prime}, P_{5} P_{5}{ }^{\prime}$ und $P_{5} P_{5}{ }^{\prime \prime}$ Kräftepaare anzubringen und das als elastisch angesehene System so zu deformiren, dass $P_{4}{ }^{\prime}$ mit $P_{4}, P_{5}{ }^{\prime}$ und $P_{5}{ }^{\prime \prime}$ mit $P_{5}$ ubereinstimmen. Das Problem des Gleichgewichts dieses Systems wird identisch sein mit dem Probleme der Ausgleichungsrechnung, sobald wir annehmen, dass die inneren Kräftepaare proportional der Winkelzunahme seien. Den Beweis dieses Satzes brauchen wir nicht durchzufuhren. Stellen wir also fest, dass das System so deformirt werden muss, dass wir ăussere Kräftepaare in den drei Zonen $P_{4} P_{4}{ }^{\prime}, P_{5} P_{5}{ }^{\prime}$ und $P_{5} P_{5}^{\prime \prime}$ anbringen, und dass diese Kräftepaare von einander unabhängig sind.

Das Problem des Gleichgewichtes eines elastischen Systems kann so behandelt werden, dass wir das Gleichgewicht zwischen den äusseren Kräften oder Kräftepaaren und den inneren Kräften oder Kräftepaaren, die aus den ausseren herstammen, einzeln betrachten.

Bevor wir aber dazu übergehen, schreiben wir folgende Zusammenslellung:

$$
\begin{array}{lll}
h_{1}=\frac{\sin \beta_{1}}{\sin \gamma_{1}} \cdot \frac{\sin \gamma_{4}}{\sin \beta_{4}} & k_{1}=\frac{\sin \beta_{1}{ }^{\prime}}{\sin \gamma_{1}{ }^{\prime}} \cdot \frac{\sin \gamma_{4}{ }^{\prime}}{\sin \beta_{4}{ }^{\prime}} & l_{1}=\frac{\sin \varepsilon_{2}}{\sin \beta_{2}} \cdot \frac{\sin \varepsilon_{4}}{\sin \beta_{4}} \\
h_{2}=\frac{\sin \beta_{3}}{\sin \gamma_{3}} & k_{2}=\frac{\sin \alpha_{3}{ }^{\prime}}{\sin \gamma_{3}{ }^{\prime}} & l_{3}=\frac{\sin \left(\varepsilon_{4}+\beta_{4}\right)}{\sin \beta_{4}} \\
h_{3}=\frac{\sin \gamma_{4}}{\sin \beta_{4}} & k_{4}=\frac{\sin \gamma_{4}^{\prime}}{\sin \beta_{4}{ }^{\prime}} & l_{4}=\frac{\sin \varepsilon_{5}}{\sin \beta_{4}{ }^{\prime}} \\
h_{5}=\frac{\sin \beta_{3}}{\sin \gamma_{3}} & k_{5}=\frac{\sin \beta_{3}{ }^{\prime}}{\sin \gamma_{3}{ }^{\prime}} & l_{6}=\frac{\sin \varepsilon_{4}}{\sin \beta_{4}} \\
h_{6}=\frac{\sin \alpha_{4}}{\sin \beta_{4}} & k_{7}=\frac{\sin \alpha_{4}{ }^{\prime}}{\sin \beta_{4}{ }^{\prime}} & l_{7}=\frac{\sin \left(\varepsilon_{5}+\beta_{4}{ }^{\prime}\right)}{\sin \beta_{4}{ }^{\prime}} \\
h_{8}=1 & k_{9}=1 & l_{10}=1,
\end{array}
$$

die die Bedingung des Gleichgewichtes ausdruckt.

Wenn zwei Kräftepaare nur in der Zone $P_{4} P_{4}^{\prime}$ angebracht werden, und 
wenn die Winkeldrehung von $P_{4}{ }^{\prime}$ gegen $P_{4}$ und in Bezug auf diese $\varphi_{1}{ }^{\prime}$ isl, werden wir für das Gleichgewicht haben

$$
\varphi_{1}^{\prime}=\left[\frac{h_{1}{ }^{2}}{g_{1}}+\frac{h_{2}{ }^{2}}{g_{2}}+\frac{h_{3}{ }^{2}}{g_{3}}+\frac{h_{5}{ }^{2}}{g_{5}}+\frac{h_{6}{ }^{2}}{g_{6}}+\frac{1}{g_{8}}\right] g_{8} c_{8}=A_{1} g_{8} c_{8} .
$$

Ebenso weun durch zwei äussere Kräftepaare, in der Zone $\boldsymbol{P}_{5} \boldsymbol{P}_{5}{ }^{\prime}$ angebracht, eine Winkeldrehung $\varphi_{2}{ }^{\prime}$ von $P_{5}{ }^{\prime}$ gegen $P_{5}$ hervorgebracht wird, wird man haben:

$$
\varphi_{2}^{\prime}=\left[\frac{k_{1}{ }^{2}}{g_{1}}+\frac{k_{2}{ }^{2}}{g_{2}}+\frac{k_{4}{ }^{2}{ }^{2}}{g_{4}}+\frac{k_{5}{ }^{2}}{g_{5}}+\frac{k_{7}{ }^{2}}{g_{7}}+\frac{1}{g_{9}}\right] g_{y} c_{9}=A_{2} g_{y} c_{9} .
$$

Und auf äbnliche Weise wird man, wenn die äusseren Kräflepaare in $\operatorname{der}$ Zone $\boldsymbol{P}_{5}$ " $\boldsymbol{P}_{5}$ wirken, in dieser Zone folgende Winkeldrehung bekommen:

$$
\varphi_{3}^{\prime}=\left[\frac{l_{1}^{2}}{g_{1}}+\frac{l_{3}^{2}}{g_{3}}+\frac{l_{4}^{2}}{g_{4}}+\frac{l_{6}{ }^{2}}{g_{6}}+\frac{l_{7}^{2}}{g_{7}}+\frac{1}{g_{10}}\right] g_{10} c_{10}=A_{3} g_{10} c_{10} .
$$

Wie man sieht, wird alles, was wir früher ausführten, auch hier ohne Einschränkung angewandt. Nun bleibt zu erwägen, was entsteht, wenn die Kräftepaare in den drei Zonen gleichzeitig wirken. Sehen wir zuvörderst, welche Winkeldrehung zwischen $P_{4}{ }^{\prime}$ und $P_{4}$ entsteht, wenn zwei Kräftepaare von der Grösse $p$ in der Zone $P_{5} P_{5}{ }^{\prime}$ wirken. Der Einfachheit halber nehmen wir an, dass nur der Winkel $a_{2}$ veränderlich sei; dann erhalten wir zuersi $g_{2} c_{2}=k_{2} p$. Nun ist es klar, dass $c_{2}$ auch dann erhalten werden kann, wenn zwei andere Krältepaare von der Grösse $q$ in der Zone $P_{4} P_{4}^{\prime}$. wirken, sobald $g_{2} c_{2}=h_{2} q$ ist, d. h. $h_{2} q=k_{2} p$.

Daraus folgt, dass die Krä́ftepaare von der Grösse $p$, in der Zone $P_{5} P_{5}{ }^{\prime}$ angebracht, dieselbe Wirkung in der Zone $P_{4} P_{4}{ }^{\prime}$ hervorbringen, wie zwei in dieser letzten Zone wirkende Kräftepaare von der Grösse $q$. Diese Wirkung ist

$$
\frac{h_{2}^{2} q}{g_{2}}=\frac{h_{1} k_{1}}{g_{1}} p .
$$

Daraus ersehen wir nebenbei, dass ein Kräftepaar, in der Zone $\boldsymbol{P}_{5} \boldsymbol{P}_{5}{ }^{\prime}$ angebracht, eine Wirkung in der Zone $\boldsymbol{P}_{4} \boldsymbol{P}_{4}{ }^{\prime}$ hervorbringt, welche mit derjenigen identisch ist, die in der Zone $P_{5} P_{5}^{\prime}$ entstchen würde, wenn dasselhe Kräftepaar in der Zone $P_{4} P_{4}{ }^{\prime}$ angebracht wäre. Dieses Reciprocitätsgeselz trägt den Namen von Maxwell.

Lassen wir nun die Annahme fallen, dass nur $a_{2}$ veränderlich sei; dann bringen die Kräftepaare von der Grösse $g_{9} c_{9}$ in der Zone $P_{5} P_{5}^{\prime}$ folgende Winkeldrehung in der Zone $\boldsymbol{P}_{4} \boldsymbol{P}_{4}{ }^{\prime}$ hervor:

$$
\varphi_{1}^{\prime \prime}=\left[\frac{h_{1} k_{1}}{g_{1}}+\frac{h_{2} k_{2}}{g_{2}}+\frac{h_{5} k_{5}}{g_{5}}\right] g_{9} c_{9}=B_{1} \cdot g_{9} c_{9} .
$$

Auf gleiche Weise erzeugen die in der Zone $P_{4} P_{4}{ }^{\prime}$ wirkenden Kräflepaare von der Grösse $g_{8} c_{8}$ folgende Winkelveränderung in der Zone $P_{5} P_{5}{ }^{\prime}$ : 


$$
\varphi_{2}^{\prime \prime}=\left[\frac{k_{1} h_{1}}{g_{1}}+\frac{k_{2} h_{2}}{g_{2}}+\frac{l_{5} h_{5}}{g_{5}}\right] g_{8} c_{8}=B_{1} \cdot g_{8} c_{8},
$$

und ebenso die Kräftepaare $g_{10} c_{10}$ in den beiden Zonen $P_{4} P_{4}{ }^{\prime}$ und $P_{5} P_{5}{ }^{\prime}$ die respectiven Veränderungen:

$$
\begin{aligned}
& \varphi_{1}{ }^{\prime \prime \prime}=\left[\frac{h_{1} l_{1}}{g_{1}}+\frac{h_{3} l_{3}}{g_{3}}+\frac{h_{6} l_{6}}{g_{6}}\right] g_{10} c_{10}=C_{1} \cdot g_{10} c_{10}, \\
& \varphi_{2}{ }^{\prime \prime \prime}=\left[\frac{k_{1} l_{1}}{g_{1}}+\frac{k_{4} l_{4}}{g_{4}}+\frac{k_{7} l_{7}}{g_{7}}\right] g_{10} c_{10}=C_{2} \cdot g_{10} c_{10} .
\end{aligned}
$$

Umgek ehrl werden die Kräftepaare $g_{8} c_{8}$ und $g_{9} c_{9}$, das eine in $P_{4} P_{4}^{\prime}$, das andere in $P_{5} P_{5}{ }^{\prime}$ angebracht, einzeln folgende Variationen in $P_{5} P_{5}{ }^{\prime \prime}$ hervorbringen :

$$
\begin{gathered}
\varphi_{3}{ }^{\prime \prime}=\left[\frac{l_{1} h_{1}}{g_{1}}+\frac{l_{3} h_{3}}{g_{3}}+\frac{l_{6} h_{6}}{g_{6}}\right] g_{8} c_{8}=C_{1} \cdot g_{8} c_{8}, \\
{\varphi_{3}{ }^{\prime \prime \prime}}^{\prime \prime}=\left[\frac{l_{1} k_{1}}{g_{1}}+\frac{l_{4} h_{4}}{g_{4}}+\frac{l_{7} k_{7}}{g_{7}}\right] g_{9} c_{9}=C_{3} \cdot g_{9} c_{9} .
\end{gathered}
$$

Nachdem dies festgestellt ist, werden wir, um die totalen Winkelveränderungen gleich den Widersprüchen $\varphi_{1}, \varphi_{2}, \varphi_{3}$ unter der Einwirkung der in den Zonen $P_{4} P_{4}{ }^{\prime}, P_{5} P_{5}{ }^{\prime}$ und $P_{5} P_{5}{ }^{\prime \prime}$ wirkenden und von einander unabhängigen Kräftepaare zu erbalten, setzen müssen:

$$
\begin{aligned}
& \varphi_{1}=\varphi_{1}^{\prime}+\varphi_{1}^{\prime \prime}+\varphi_{1}^{\prime \prime \prime} \\
& \varphi_{2}=\varphi_{2}^{\prime}+\varphi_{2}^{\prime \prime}+\varphi_{2}^{\prime \prime \prime} \\
& \varphi_{3}=\varphi_{3}^{\prime}+\varphi_{3}^{\prime \prime}+\varphi_{3}^{\prime \prime \prime} .
\end{aligned}
$$

Wenn wir voriges berucksichtigen, erhallen wir folgende Gleichungen:

$$
\begin{aligned}
& A_{1} g_{8} c_{8}+B_{1} g_{9} c_{9}+C_{1} g_{10} c_{10}=\varphi_{1}, \\
& B_{1} g_{8} c_{8}+A_{2} g_{9} c_{9}+C_{1} g_{10} c_{10}=\varphi_{2}, \\
& C_{1} g_{8} c_{8}+C_{2} g_{9} c_{9}+A_{3} g_{10} c_{10}=\varphi_{3},
\end{aligned}
$$

welche dazu dienen, die Verbesserungen $c_{8}, c_{9}, c_{10}$ zu bestimmen.

Dann lässt sich folgendes berechnen:

$$
\begin{aligned}
& g_{1} c_{1}=h_{1} \cdot g_{8} c_{8}+k_{1} \cdot g_{9} c_{9}+l_{1} \cdot g_{10} c_{10} \text {, } \\
& g_{2} c_{2}=h_{2} \cdot g_{8} c_{8}+k_{2} \cdot g_{9} c_{9} \text {, } \\
& g_{3} c_{3}=h_{3} \cdot g_{8} c_{8}+l_{3} \cdot g_{10} c_{10} \text {, } \\
& g_{4} c_{4}=\quad k_{4} \cdot g_{9} c_{9}+l_{4} \cdot g_{10} c_{10} \\
& g_{5} c_{5}=h_{5} \cdot g_{8} c_{8}+k_{5} \cdot g_{8} c_{9} \text {, } \\
& g_{6} c_{6}=h_{6} \cdot g_{8} c_{8}+l_{6} \cdot g_{10} c_{10} \text {, } \\
& g_{7} c_{7}= \\
& k_{7} . g_{9} c_{0}+l_{7} . g_{10} c_{10} \text {. }
\end{aligned}
$$

Rom, Mai 1894. 